\title{
Ayuda a la clasificación y priorización en la evacuación de bajas de combate. Ayuda al proceso asistencial. Proyecto e-Safe'Tag
}

\author{
del Real Colomo A. ${ }^{1}$, Setién Dodero F. ${ }^{2}$, Moreno Caravaca A. ${ }^{3}$, Hernández Abadía de Barbara A. ${ }^{4}$
}

Sanid. mil. 2014; 70 (4): 288-292; ISSN: 1887-8571

\begin{abstract}
RESUMEN
La asistencia sanitaria a las bajas de combate es el objetivo final de la Sanidad Militar. La implantación de protocolos asistenciales como el TCCC (Tactical Combat Casualty Care) ha ayudado a mejorar dicha asistencia, pero el personal sanitario sigue encontrándose con problemas (falta de acceso a Historia Clínica, dificultad en la transferencia del herido). El Proyecto e-SafeTag se ha diseñado para ayudar al personal sanitario proporcionándole información clínica de la baja, y un material (PDA y radio) de apoyo en el proceso asistencial y que permite la transmisión de la información de forma automática.
\end{abstract}

PALABRAS CLAVE: e-SafeTag, Baja de combate, TCCC, Control de bajas, Historia clínica.

Assistance to the classification and prioritization in the evacuation of combat casualties. Assistance to the healthcare process. e-SafeTag project

SUMMARY: health care for combat casualties is the ultimate aim of the military health. The implementation of care protocols as TCCC (Tactical Combat Casualty Care) has helped improve that care, but the medical staff is incountering problems (lack of access to clinical history, difficulty in transferring the wounded). The e-SafeTag Project is designed to assist health workers providing clinical information of the casualty, and item (PDA and radio) support in the care process and allows the transmission of information automatically. KEYWORDS: e-SafeTag, Combat casualty, TCCC, Control casualty, Medical history.

\section{INTRODUCCIÓN}

La asistencia sanitaria a las bajas de combate es el objetivo final de la Sanidad Militar.

Actualmente se ha avanzado mucho en esta asistencia gracias a la implantación de protocolos asistenciales como el TCCC (Tactical Combat Casualty Care), pero el personal sanitario encargado de la asistencia inicial a dichas bajas sigue encontrándose con problemas como el desconocimiento del historial médico del herido, y la transmisión de toda la información que se genera durante la asistencia hasta las instalaciones de tratamiento médico donde se recibirá la baja finalmente.

El Proyecto e-SafeTag se ha diseñado para ayudar al personal sanitario proporcionándole información clínica de la baja, que va codificada en un chip adherido a la chapa de identificación, y un material (PDA y radio) que le sirve de quía en la asistencia, le permite recopilar los datos clínicos del paciente (ECG, frecuencia cardíaca, frecuencia respiratoria, saturación arterial de oxígeno....), y la transmisión de los mismos de forma inmediata a

\footnotetext{
${ }^{1}$ Cte. Médico. Hospital Central de la Defensa Gómez Ulla. Servicio de Telemedicina. ${ }^{2}$ Ingeniero de Telecomunicaciones. IGESAN.

3 Brig. CGEA. Hospital Central de la Defensa. Servicio de Fotografía.

${ }^{4}$ Tcol. Médico. Dirección de Sanidad del Ejército de Tierra.
}

Dirección para correspondencia: Antonio del Real Colomo. Servicio de Telemedicina. Hospital Central de la Defensa Gómez Ulla. Glorieta del Ejército nº 1, 28047 Madrid. España. Tel.-914222073. telemedicina@oc.mde.es

Recibido: 20 de mayo de 2014

Aceptado: 8 de julio de 2014 los centros de Control de Misión y de Control de Bajas, así como facilita la transferencia del herido entre profesionales sanitarios.

El presente proyecto, e-SafeTag, surge como consecuencia del Acuerdo de Cooperación Industrial entre el Consorcio Iveco Oto Melara -CIO- y el Ministerio de Defensa Español, asociado al suministro de vehículos CENTAURO para el Ejército de Tierra.

Esta compra de vehículos lleva asociado un proyecto válido para promover el desarrollo industrial español según la política de Cooperación Industrial Española.

El proyecto cumple con la finalidad de "promover la participación en los programas multinacionales o en los programas de cooperación" del sector industrial nacional. (Figura 1).

El objetivo principal del proyecto e-SafeTag es desarrollar una aplicación de atención médica innovadora que venga a apoyar el trabajo de los médicos de las Fuerzas Armadas españolas, cuyo escenario de actuación se sitúa sobre el terreno, ya sea en misiones de paz, en zonas de conflicto o en situaciones de emergencia.

Esta aplicación se integra perfectamente dentro del "Tactical Combat Casuality Care" (TCCC), que es el estándar de la OTAN que define los procedimientos para la atención de bajas en combate.

El contexto de uso de la aplicación es en la segunda y tercera fases asistenciales marcadas en el TCCC, es decir en los cuidados "tácticos" o en el propio campo de batalla (TFC) y en la fase de Evacuación de la baja (CCEC). Fuera de este contexto no está indicado hacer nada mas que aquellas maniobras que salvan vidas, por lo tanto no se debe considerar su uso en la fase de cuidados bajo el fuego (CUF) del TCCC. 


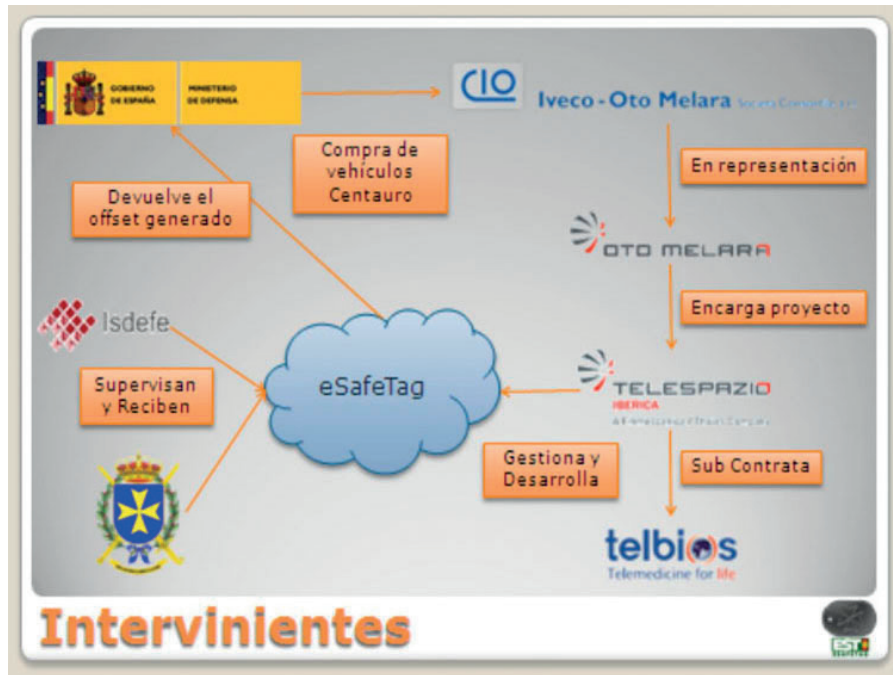

Figura 1.

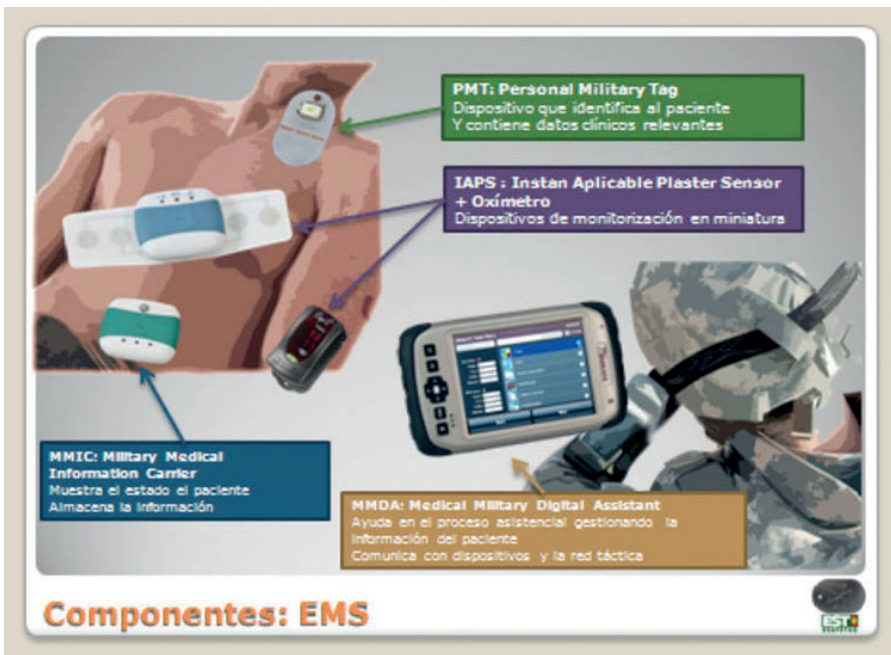

Figura 2.

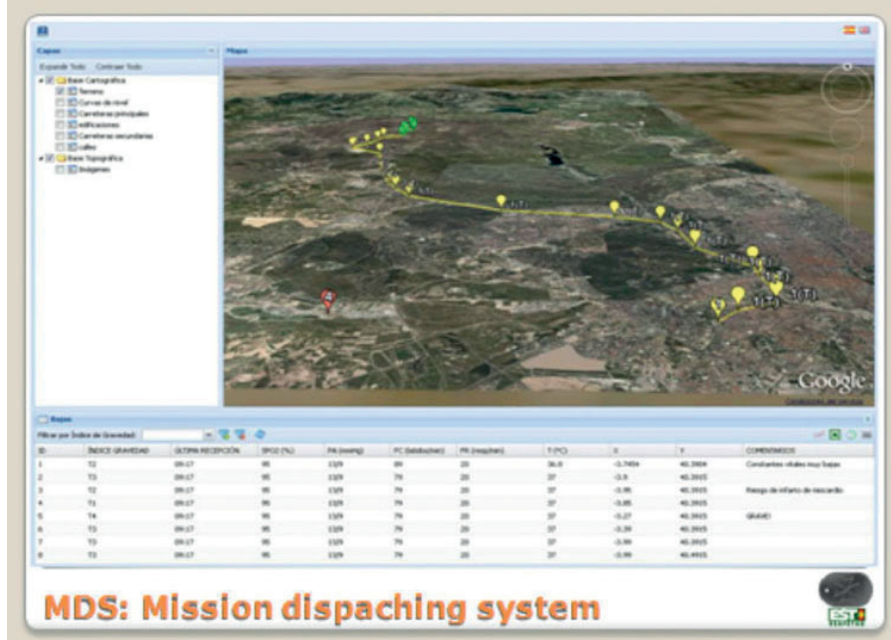

Figura 3.
Se ideó un sistema de dispositivos que permitiesen apoyar al sanitario que atiende la baja sobre el terreno, de forma que dispusiese de mucha más información que le ayudase en la toma de decisiones, y automatizase algunos cálculos sobre la prioridad en la evacuación del herido en combate.

Este proyecto se ha visto favorecido por la incorporación de nuevos equipos de comunicaciones tácticas a nuestras Fuerzas Armadas. Estos equipos permiten crear redes "ad Hoc" entre ellos de tal manera que puedan soportar las comunicaciones sanitarias que se generan con los dispositivos del proyecto e-SafeTag sin comprometer el resto de comunicaciones en zona.

\section{DISPOSITIVOS}

Los dispositivos que forman el sistema e-SafeTag se distribuyen en dos grandes grupos:

- On-field EMS (Emergency Monitoring System): (Figura 2) consta de aquellos dispositivos, aplicaciones y comunicaciones que se despliegan con el asistente sanitario y que permiten:

- Acceso al historial clínico del herido.

- Monitorización de las constantes vitales en tiempo real.

- Soporte a la evaluación médica y toma de decisión del médico en campaña, así como la transferencia del herido al Role superior.

- Base-field MDS (Mission Dispatching System): (Figura 3) aquellos dispositivos integrados en los centros de control de misión y de control de bajas. Permite conocer la ubicación de los heridos, su distribución tanto en número como por gravedad, e histórico de las evacuaciones, aportando un punto de referencia para la toma de decisiones en Role 2,3 y superior.

\section{DESARROLLO DEL PROYECTO}

\section{1. $^{a}$ Fase de desarrollo}

El Proceso asistencial con el sistema e-SafeTag se inicia una vez retirada la baja de la zona de fuego.

Realizado un triaje clínico inicial, se pasa a leer la información del paciente que va incluida en la chapa de identificación mediante un dispositivo de almacenamiento electrónico que contiene los datos mínimos de salud exigidos por la OTAN para todo su personal. (Identificación, alergias, estado vacunal, enfermedades y cirugías previas...) (Figura 4).

Lo que nos permite iniciar las maniobras de resucitación si fuesen necesarias o la estabilización de la baja con la seguridad de conocer los datos mínimos de salud de la misma.

Una vez realizado el control de hemorragias exanguinantes, valoración de la vía aérea, la respiración y la circulación (C$\mathrm{ABC}$ de la asistencia al politraumatizado) es cuando pasamos a seguir las indicaciones del sistema rellenando los datos de Triaje o clasificación. Para ello se completan los formularios basados en la Escala de Coma de Glasgow modificada añadiendo la frecuencia respiratoria. La tensión arterial se puede tomar 


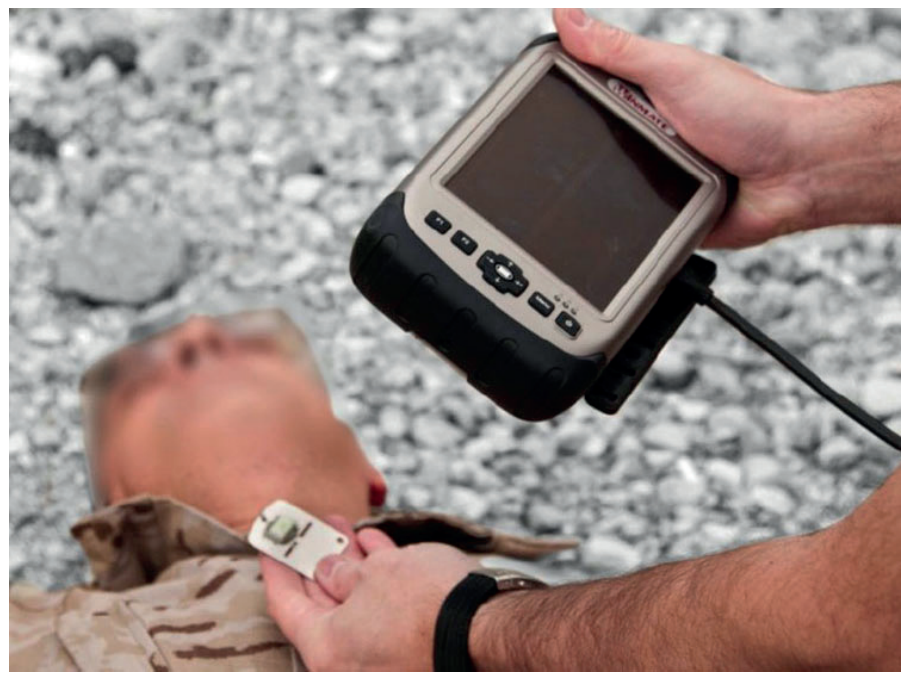

Figura 4.

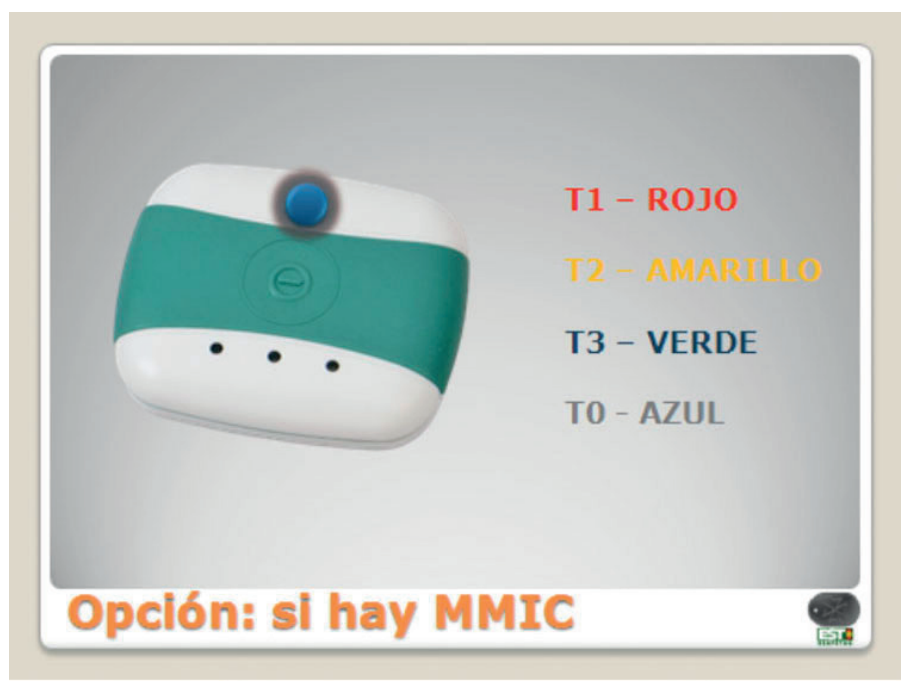

\section{Figura 5.}

explorando la existencia de pulso radial o carotídeo. Esto nos ofrecerá una clasificación con un índice de gravedad según el Trauma Score Revisado que nos informará si el paciente es un grupo T1-ALFA-ROJO, T2-BRAVO-AMARILLO, T3CHARLIE-VERDE, o T4-DELTA-AZUL. (Elegimos el color azul ya que el negro es muy difícil de realizar con las luces que se utilizarán en el dispositivo de almacenamiento de información MMIC) (Figura 5).

De forma inmediata esta información, en el momento es enviada por vía radio táctica al centro de control de misión, donde tendrán información fiel de lo que le está ocurriendo a la baja de combate.

Además el sistema permite adquirir datos útiles del paciente mediante la utilización de los sensores incluidos en el mismo: tira de ritmo, frecuencia cardiaca, frecuencia respiratoria y saturación de Oxígeno. Estos datos se remiten de forma inmediata a los centros de control de bajas y de misión (Figuras 6-8).

Como es lógico, el sistema permite cumplimentar la tarjeta de cuidados tácticos TCCC de manera que se puede ampliar la información clínica añadiendo sobre un esquema corporal las

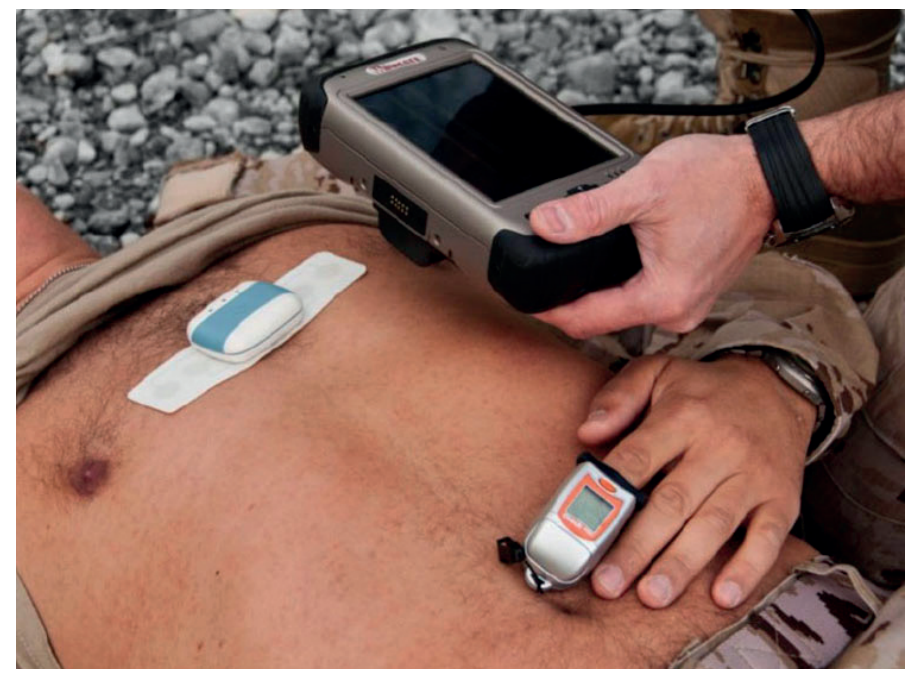

Figura 6.

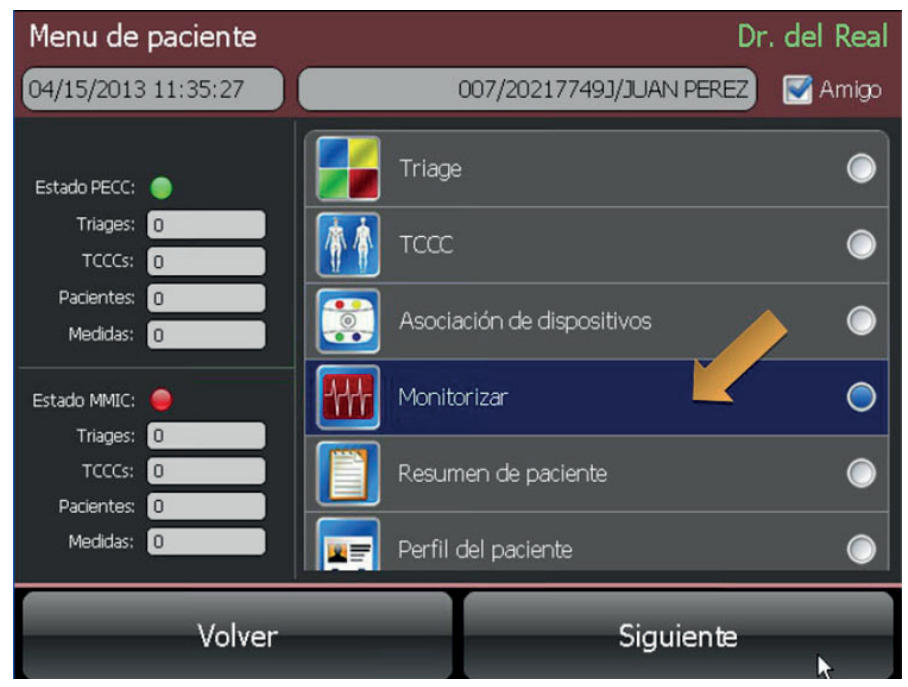

Figura 7.

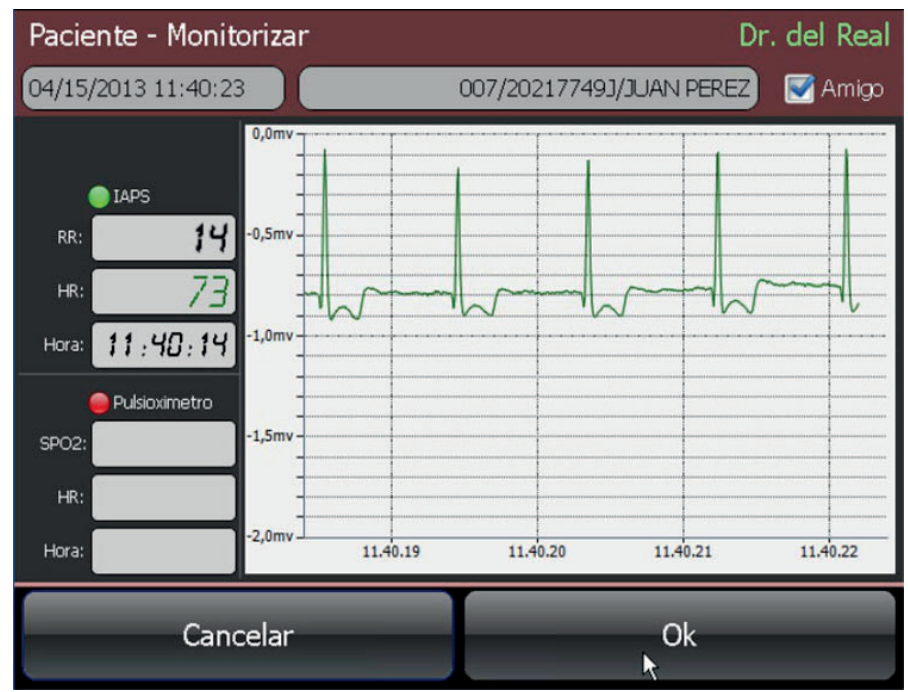

Figura 8. 


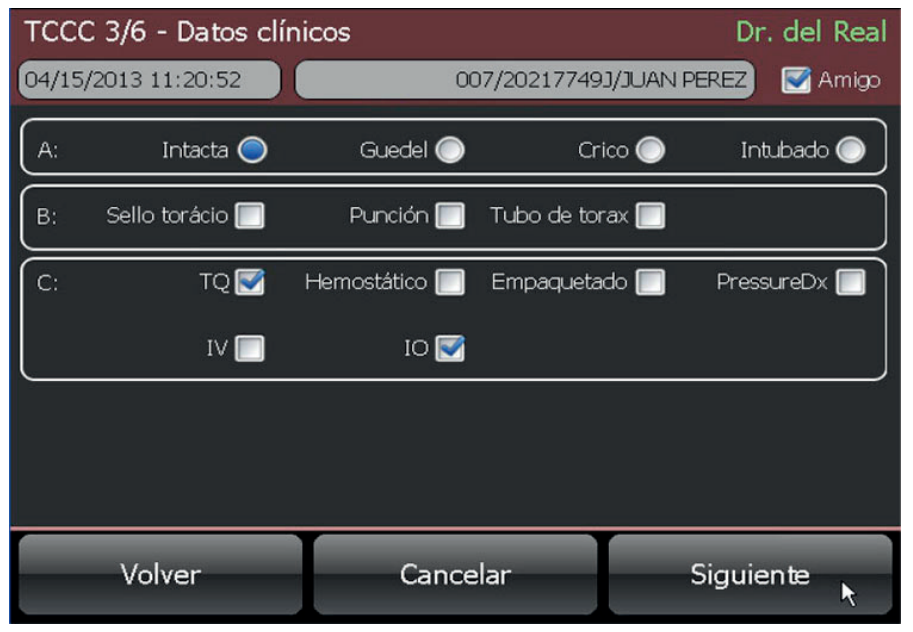

Figura 9.

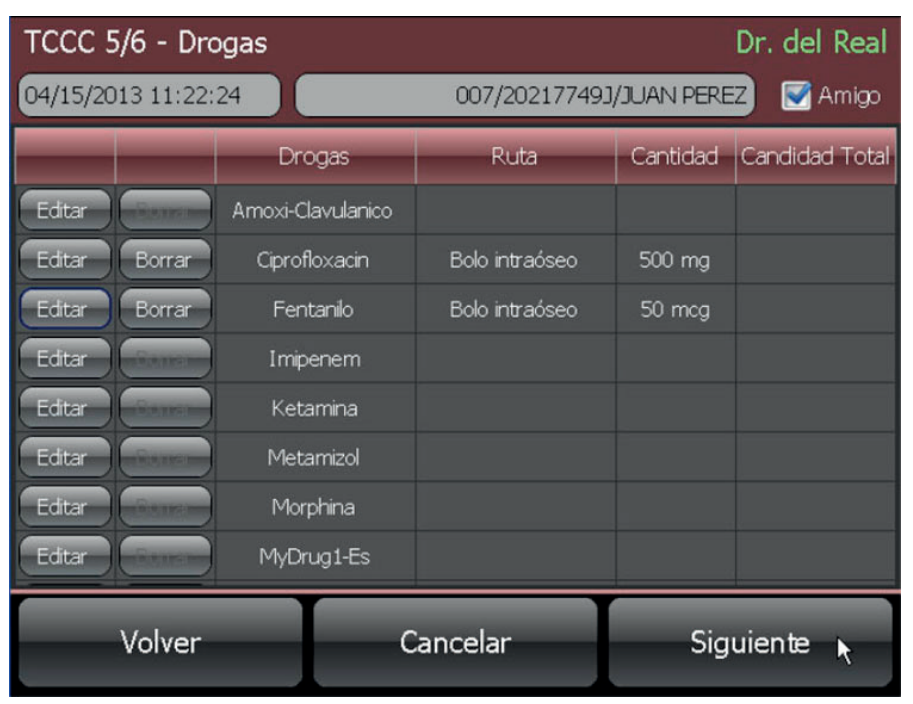

Figura 10.

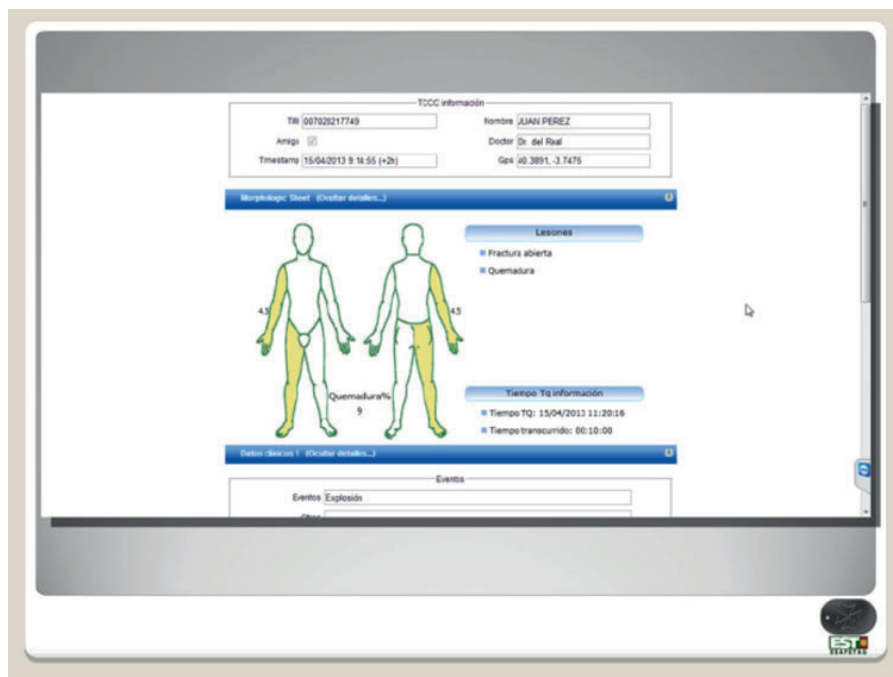

Figura 11 . zonas lesionadas y la patogenia de la lesión, la aplicación de torniquete y la duración de la misma, y la evaluación según el $\mathrm{C}$ - $\mathrm{ABC}$ marcando las actuaciones que se realizan sobre la vía aérea, la respiración y la circulación (Figura 9).

También permite registrar los fluidos y medicación que se administra al paciente y las dosis establecidas para cada fármaco y la vía de administración, y el envío inmediato de estos datos. (Figuras 10, 11).

El trabajo en condiciones tácticas puede implicar en gran parte de los casos, la transferencia de la baja entre distintos equipos sanitarios, es decir, la célula de estabilización puede verse obligada a transferir la baja al equipo MEDEVAC. Todos conocemos la dificultad que implica una transferencia rápida en las condiciones en que se suele realizar (condiciones tácticas de cierta inseguridad, ruido de los rotores...) aunque seamos absolutamente sistemáticos en la descripción de las lesiones y actuación sobre las mismas.

El sistema e-SafeTag puede ayudar en esta circunstancia, ya que al tener toda la información almacenada en el dispositivo MMIC puede transferirse rápidamente hasta un nuevo sistema de lectura MMDA sin pérdida de información, y recuperarse simplemente eligiendo en el menú inicial la opción de abrir desde MMIC. También, con la opción Resumen de paciente se puede ver de una forma rápida y en un solo vistazo toda la situación (Figuras 12-14).

Durante el tiempo de evacuación, los protocolos de asistencia exigen la reevaluación continua de la baja. Cada vez que se realiza una reevaluación el sistema genera un nuevo registro temporal con los datos clínicos y de geolocalización.

Esto permite por tanto el tratamiento posterior de los datos adquiridos, al almacenarse los mismos en forma de registro histórico. También se almacena un trazo sobre la cartografía de la zona que indica la ruta por la que ha sido evacuada la baja, de tal manera que se puede hacer un estudio sobre la situación de la baja en cada uno de los puntos por lo que ha pasado la misma.

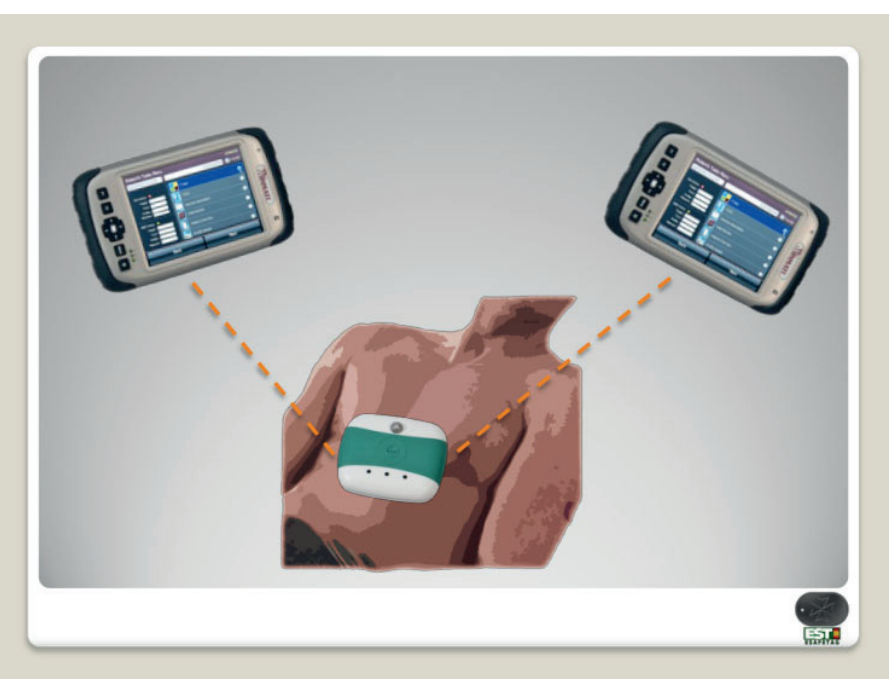

Figura 12. 


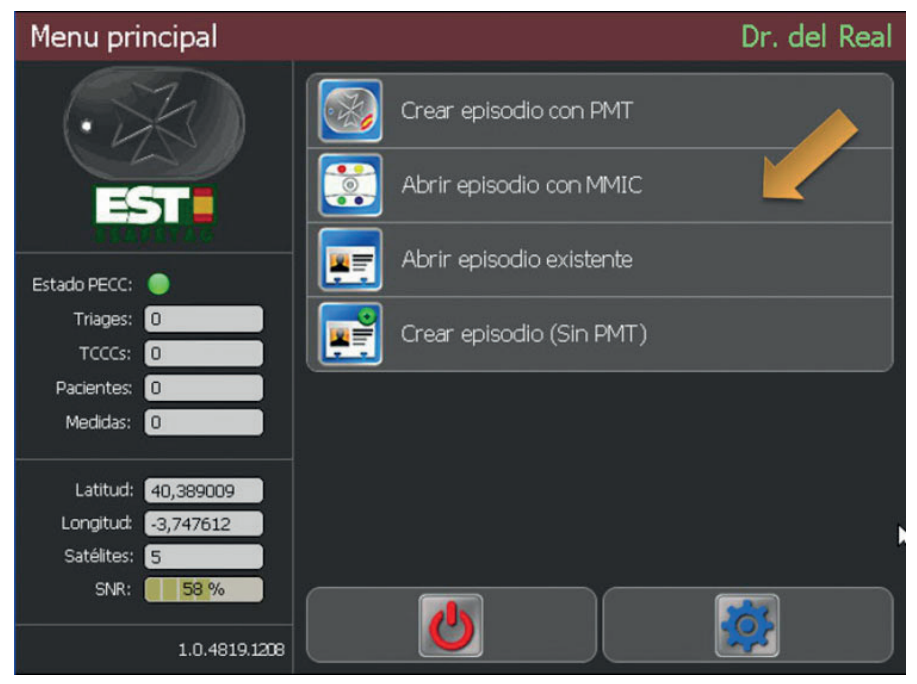

Figura 13.

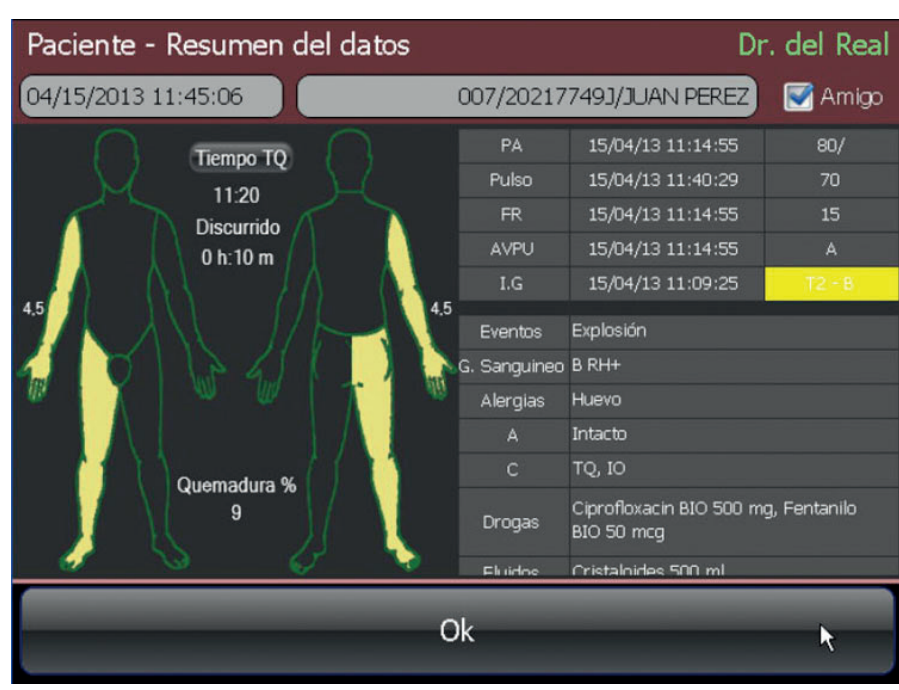

Figura 14.

\section{2. ${ }^{\mathrm{a}}$ Fase de desarrollo}

Tras la primera evaluación del Proyecto, pensamos que determinados grupos de edad dentro del personal sanitario podrían mostrar ciertos reparos a la hora de utilizar unos conceptos tan absolutamente novedosos en la asistencia a la baja.

Por ello, como transición previa al uso de todos los dispositivos electrónicos o bien de forma complementaria a los mismos se puede introducir el uso de unos bolígrafos que nos permiten seguir trabajando en la forma clásica, pero que envían toda la información a los centros de control en formato similar al que lo hacen los dispositivos informáticos.

Esto es posible mediante el uso de los dispositivos ANNOTO, bolígrafos que escribiendo sobre una plantilla de papel impreso son capaces de adquirir toda la información y enviarla utilizando los mismos sistemas de comunicaciones tácticas. La plantilla se imprime con una impresora normal, sobre papel normal, que se convierte al imprimirlo en una plantilla específica para cada dispositivo. (Figuras 15, 16).

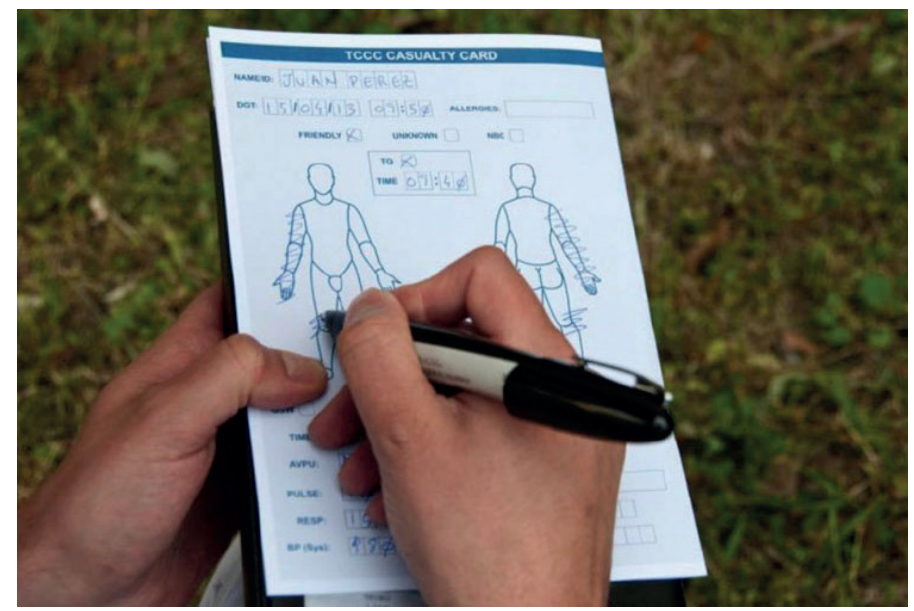

Figura 15.

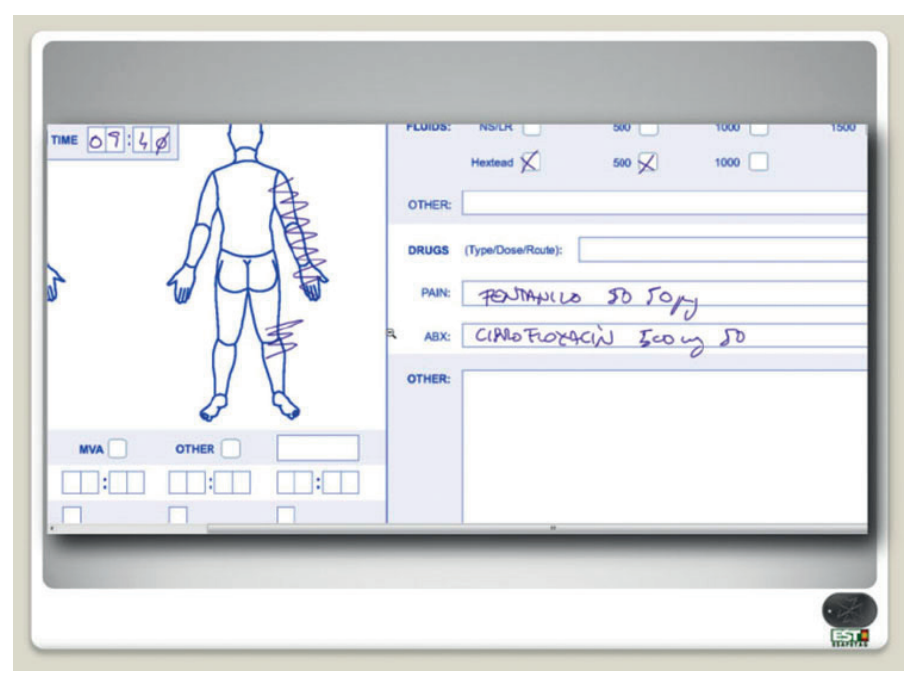

Figura 16.

Con estas aplicaciones consideramos que se mejora la asistencia a la baja de combate, ya que se optimiza el proceso de clasificación y priorización en la evacuación. Asimismo se presta un apoyo valioso al sanitario que realiza la asistencia al proporcionarle las listas de fármacos, sueros, etc, y las dosis correspondientes de los mismos, teniendo solamente que elegir entre ellos.

Falta desarrollar por el momento un algoritmo matemático que permita la medida de la Presión Arterial, de forma indirecta, a partir de los dispositivos que ya están integrados en el sistema, para no tener que añadir ninguno más. Vamos por buen camino en este sentido ya que estamos desarrollando un sistema que mide la P.A a partir de la diferencia de tiempo entre la onda $\mathrm{R}$ del complejo QRS del EKG, y el inicio de la onda de presión del pulso, con el mismo error y desviación típica que un manómetro de mercurio.

Por otra parte, en fechas próximas realizaremos las pruebas de campo de todo el sistema para hacer la validación sobre el terreno, y la adición del apoyo por voz a todas las decisiones además de la transmisión de los datos médicos, de forma que se pueda interactuar no solo en el sentido de escalón inferior hacia el superior, sino desde los centros de control hasta donde se encuentra la baja, con los que los sanitarios sobre el terreno se podrán sentir aún más apoyados 